\title{
Evaluative conditioning induces changes in sound valence
}

\author{
Anna C. Bolders ${ }^{1 *}$, Guido P. H. Band ${ }^{1,2}$ and Pieter Jan Stallen ${ }^{1}$ \\ 1 Cognitive Psychology Unit, Institute of Psychology, Leiden University, Leiden, Netherlands \\ ${ }^{2}$ Leiden Institute for Brain and Cognition, Leiden, Netherlands
}

\section{Edited by:}

Eduardo B. Andrade, University of

California Berkeley, USA

\section{Reviewed by:}

Joel Cohen, University of Florida, USA

Stijn M. J. Van Osselaer, Erasmus

University, Netherlands

\section{*Correspondence:}

Anna C. Bolders, Cognitive

Psychology Unit, Institute of

Psychology, Faculty of Social and

Behavioral Sciences, Leiden

University, P.O. Box 9555, 2300 RB

Leiden, Netherlands.

e-mail: abolders@fsw.leidenuniv.nl

Through evaluative conditioning (EC) a stimulus can acquire an affective value by pairing it with another affective stimulus. While many sounds we encounter daily have acquired an affective value over life, EC has hardly been tested in the auditory domain. To get a more complete understanding of affective processing in auditory domain we examined EC of sound. In Experiment 1 we investigated whether the affective evaluation of short environmental sounds can be changed using affective words as unconditioned stimuli (US). Congruency effects on an affective priming task for conditioned sounds demonstrated successful EC. Subjective ratings for sounds paired with negative words changed accordingly. In Experiment 2 we investigated whether extinction occurs, i.e., whether the acquired valence remains stable after repeated presentation of the conditioned sound without the US. The acquired affective value remained present, albeit weaker, even after 40 extinction trials. These results provide clear evidence for EC effects in the auditory domain. We will argue that both associative as well as propositional processes are likely to underlie these effects.

Keywords: evaluative conditioning, affective priming, extinction, valence, environmental sounds, auditory processing

\section{INTRODUCTION}

The smell of kerosene, the sound of gulls, and the taste of sea water, although affectively ambivalent, can all attain a positive connotation following a pleasant beach vacation. Likewise, it has been demonstrated with evaluative conditioning (EC) studies that affective evaluation can be modified by pairing the evoking stimulus with affective stimuli (De Houwer et al., 2001; Hofmann et al., 2010). Although the effect has been demonstrated in a variety of conditions and modalities, the change in affective valence of sounds through EC has been scarcely studied (Hofmann et al., 2010, but see Bliss-Moreau et al., 2010 for a recent exception). To investigate whether this effect generalizes to the auditory domain, in the current study short everyday sounds were used in an EC procedure. We tested for the occurrence of effects both on a subjective (explicit) and on an implicit measure. In addition it was investigated whether the acquired evaluation remained stable over repeated presentations of the conditioned stimulus (CS) alone, i.e., whether extinction occurred.

Evaluative conditioning can be regarded as a form of conditioning that is specifically concerned with a change in valence of a stimulus (Levey and Martin, 1975; De Houwer et al., 2001; Hofmann et al., 2010). In a typical EC experiment, neutral stimuli are selected, which will function as CS. Then, during an acquisition phase, each stimulus is repeatedly paired with a unconditioned stimuli (US), a stimulus that has a clear positive or negative valence. Finally, the evaluation of the CS is measured in order to establish whether it has changed due to the EC procedure. Importantly, other factors that could change the evaluation, such as mere exposure (Zajonc, 1968) of a stimulus should be controlled for (De Houwer, 2008). A large body of research using the EC procedure and controlling for these factors has demonstrated that EC is a genuine effect that occurs with various types of stimuli under various conditions (De Houwer et al., 2001, 2005; De Houwer, 2007).

Most studies on EC have used self-report to measure whether the valence of the CS has changed. This way of assessing how people evaluate the stimuli gives them ample time to think about this evaluation and exert control over it. In daily life, however, behavior often arises spontaneously and is therefore guided by evaluations of stimuli in a relatively automatic, rather than controlled manner (Zajonc, 1980; De Houwer, 2003, 2006; Fazio and Olson, 2003). To probe automatic evaluation, implicit measures can be used, such as in a speeded categorization task (Gawronski, 2009), where no explicit reference is made to the valence of the stimulus of interest, but where it does affect performance. These measures are better suited to predict spontaneous behavior (De Houwer, 2003, 2006; Fazio and Olson, 2003) since the outcome of implicit measures should, according to the normative description of De Houwer et al. (2009), reflect evaluations in an automatic way (De Houwer, 2006; De Houwer et al., 2009).

In addition, implicit measures are less likely to be influenced by demand characteristics; cues that signal the research hypothesis (Orne, 1962), than self-report measures (Hermans et al., 2003b). In many EC studies it is obvious to the participant that a change in evaluation of the CS is expected, especially when only few CS and US are used (Mitchell et al., 2003; Field, 2005). Responses on implicit measures are generally assumed to be difficult to control and this makes them well suited to circumvent the demand problem (De Houwer, 2003; Gawronski, 2009). It has to be noted that recent studies have cast doubt on the full uncontrollability of responses on implicit measures. Under specific conditions, for example when control strategies are provided, participants are able 
to exert deliberate control on responses to the affective priming task (APT); a widely used implicit measure (Degner, 2008; TeigeMocigemba and Klauer, 2008). In line with the perspective of Gawronski (2009) we assume that implicit measures may indeed be controllable, but much less than subjective ratings.

To assess affective evaluation in the current study we employed an APT. In an APT, a participant is briefly presented with prime stimuli, which potentially trigger an implicit evaluation. After a very short interval the prime stimulus is followed by a positive or negative target stimulus. The participant is asked to quickly classify the valence of the target by pressing one of two response keys. Reaction time (RT) and accuracy effects in the APT reflect the response tendency induced by the prime. Responses will be faster and have higher accuracy when prime and target are of the same valence (congruent) than when they are of opposite valence (incongruent). Thus, the evaluation of the prime can be inferred from these facilitation or interference effects (De Houwer et al., 2009).

Several previous studies have applied the APT to demonstrate EC effects by using the conditioned stimuli, e.g., human faces (Hermans et al., 2003a), pseudo words (Aguado et al., 2005), and vocal signals (Bliss-Moreau et al., 2010) as prime. Likewise, conditioned sounds will be employed as primes in an APT in the present study. Congruence effects on this task demonstrate that the acquired evaluation of the sounds manifest relatively automatically. To our knowledge, the current study is the first to use the APT with environmental sounds. In contrast to visual information as mostly used in the APT, auditory information is not instantaneous, but builds up over time. The APT effect is known to be sensitive to timing parameters of the prime and target (Hermans et al., 2001, 2003b), which may thus differ for visual and auditory information. Therefore, we included trials with pre-rated positive and negative sounds of similar length as the conditioned sounds. If affective priming effects are found with these sounds, it validates the use of APT as a measure of sound evaluation (De Houwer et al., 2009). In addition this allows for a comparison of priming effects of the conditioned stimuli with those of the pre-rated positive and negative sounds.

\section{EXPERIMENT 1: EC EFFECT}

The experimental procedure of the first experiment consisted of several phases. During the CS selection phase participants rated a set of sounds and the sounds rated most neutrally were selected as CS. These sounds were paired with positive, negative, or neutral words (US) during the conditioning phase and were used as primes in an APT in the affective priming phase. In addition, ratings of the CS were acquired during the post EC rating phase. Given previous findings of EC effects we expected more positive subjective ratings for sounds paired with positive than with neutral US and more negative ratings for sounds paired with negative than with neutral US. Likewise, we expected to find priming effects due to the changed liking of the CS on the APT.

\section{MATERIALS AND METHODS Participants}

Twenty-four Dutch speaking participants (one male, five left handed) ranging in age from 18 to 29 years $(M=21.4$ years,
$\mathrm{SD}=2.6$ years) with self-reported normal hearing took part in the experiment for which they received course credits or $€ 6.50$. All participants signed an informed consent form before the start of the study. The study was approved by the ethical committee of the Institute of Psychology of Leiden University.

\section{Materials}

Apparatus. Stimulus presentation was controlled by e-prime ${ }^{\circledR}$ 2.0 (Schneider et al., 2002) using a computer with a CRT screen $(100 \mathrm{~Hz}$ refresh rate, $1024 \times 768$ resolution). Sounds were binaurally presented through insert earphones (Etymotic ER-4B microPro) which provide $35 \mathrm{~dB}$ external noise attenuation. Responses were made on a QWERTY keyboard and by using a mouse.

Sounds. Sounds came from various sources and had all been used in previous research (Fabiani et al., 1996; Bradley and Lang, 2007; Gygi et al., 2007). For all sounds, digital sound properties were standardized $(44 \mathrm{kHz}, 16 \mathrm{bit}$, mono). The length of the sounds adopted from Fabiani et al. (1996) was left unchanged, while sounds from the International Affective Digitized Sounds (IADS, Bradley and Lang, 2007) and Gygi et al. (2007) were cut to short clips at natural breaks in the sound or with $5 \mathrm{~ms}$ ramped offset to minimize clicks. The sounds reflected events from various sources (e.g., animals, music, nature, human non-verbal vocalizations, mechanical machines, and computers). All sounds were rated previously in a separate sound rating study on a computeradministered pictorial nine-point self assessment-manikin valence scale (SAMv; Bradley and Lang, 1994) ranging from very unpleasant (1) to very pleasant (9) $(N=15$, age: $18-30$, normal hearing, Dutch speaking).

A set of 18 sounds with ratings close to neutral [valence: $M=4.94, \mathrm{SD}=0.50$, duration ( $\mathrm{ms}): M=312, \mathrm{SD}=70]$ was used for the CS selection phase. Based on individual SAMv ratings made during the CS selection phase of the present experiment, six sounds rated closest to neutral were selected by the computer program for further use during the experiment. Three of these sounds were randomly assigned to serve as CS and subsequently as primes for the APT. Two additional pre-rated sounds served as primes in the semi-experimental validation trials of the APT; the sound of a bird chirping which was rated as positive and the sound of a gunshot which was rated as negative. Finally, four sounds were selected to serve as practice sounds in the CS selection phase and in the APT [Valence: $M=5.30, \mathrm{SD}=1.43$, duration (ms): $M=304$, $\mathrm{SD}=118]$. More details about the duration, SAMv ratings, and the source of all used sounds can be found in Table A1 in the Appendix.

Words. All words used in the EC phase and the affective priming phase were chosen from the word norms of Hermans and De Houwer (1994) that provide ratings of 740 Dutch words on affect $(1=$ very negative $7=$ very positive $)$ and familiarity $(1=$ not familiar at all, $7=$ very familiar . All words were presented in a black 18 point Courier New Font on a white background.

Two sets (set A and set B) each consisting of 15 nouns were selected to serve as US. Each set consisted of subsets of five positive, five negative, and five neutral words. Words from one of the two sets were used as US. This was counterbalanced across participants. The subsets of the same valence in set A and set B were 
matched for affect, affective extremity (extent to which the rating deviated from neutral score 4), familiarity, and word length (in characters). The positive, negative, and neutral word sets differed in rated affect. Positive and negative words sets were matched for affective extremity while ratings of neutral words were lower than of positive and negative sets on this measure. The three word sets were matched for familiarity and word length. Means for affect, affect extremity, familiarity, and word length per valence category are shown in Table 1. Specifications of each word can be found in Table A2 in the Appendix.

Twelve positive and 12 negative adjectives were selected to serve as targets in the APT. Positive and negative word sets differed on affect, but were matched for affective extremity, familiarity, and word length. Specifications of individual words can be found in Table A3 in the Appendix.

\section{Procedure}

Upon arrival, participants were guided to a dimly lit individual test cubicle and were seated at $50 \mathrm{~cm}$ from the computer monitor, where further instructions were provided. In order to reduce demand characteristics, the study was introduced as a study on the influence of sounds on memory for words.

CS selection phase. Participants were presented with 18 sounds and were asked after every sound to rate how pleasant they experienced it by clicking on a nine-point SAMv scale. It was stressed that they should rely on their direct, personal experience, and that there were no correct or wrong answers. Before the start of the CS selection task, four practice sounds were rated to familiarize the participants with the type of sounds that would be presented and to the way of rating them. The sound ratings collected during the selection phase were used as baseline measures and to select sounds to serve as CS.

Evaluative conditioning phase. Participants were instructed that they would be presented with words and sounds to which no direct response was required, but that they should allow the words and sounds to affect them. They were told that this would be important for a later part of the study.

During the EC procedure each sound was repeatedly paired with words of the same valence type (positive, negative, or neutral). Three sounds were paired with supra-optimally presented words (presented long enough to allow for conscious awareness) and the three remaining sounds were paired with sub-optimally presented words (presented short enough to make conscious awareness unlikely for most participants). For each sound five different words were used as US, which were presented four times, resulting in 20 trials per sound. The order of the trials and conditions was randomized.

A trial started with a warning text ("attention!") which was presented for $2 \mathrm{~s}$, immediately followed by the presentation of a CS sound, during which a fixation cross appeared on the screen. The US word replaced the fixation cross directly after the offset of the sound and remained on the screen for $540 \mathrm{~ms}$. The inter-trial interval was $1 \mathrm{~s}$. In the trials with sub-optimal US presentation, the fixation cross was replaced by a letter string as forward mask for $250 \mathrm{~ms}$, followed by $40 \mathrm{~ms}$ US word presentation and thereafter the same letter string as backward mask of $250 \mathrm{~ms}$. No EC effects were found on any of the measures for sounds paired with subliminally presented words, which is in line with the results of the metaanalysis by Hofmann et al., 2010. The discussion of the absence of the EC effect for subliminal US presentation falls outside the scope of the main research questions in this paper. Therefore, in the present paper only parameters and results related to the supraoptimally presented words and sounds paired with these words are discussed in detail.

Affective priming phase. Before the start of the APT the participants were told that they would again hear a sound followed by a word. They were instructed to judge whether the word was positive or negative by pressing the z-key on the keyboard with their left index finger or the m-key on the keyboard with their right index finger. The assignment of valence to the keys was counterbalanced across participants. It was stressed that they should concentrate on the judgment of the word and not on the sound and that they should try to give their response as fast and accurately as possible.

Each trial started with a fixation cross presented in the middle of the screen for $1900 \mathrm{~ms}$, which was followed by a blank screen. The prime sound was presented $100 \mathrm{~ms}$ after the onset of the blank screen. The target word appeared on the screen $300 \mathrm{~ms}$ after sound onset and remained there until the participant responded or $2000 \mathrm{~ms}$ had elapsed. A blank screen was presented during the response-stimulus interval of $2000 \mathrm{~ms}$ that followed. Timing of prime, target, response window, and response-stimulus interval were patterned after previous studies using APT to measure acquired evaluation (Hermans et al., 2003b; Aguado et al., 2005).

The APT consisted of 192 trials in which eight prime sounds (three CS sounds that had been supra-optimally conditioned, three

Table 1 | Means (SD) for affect, affect extremity, familiarity, and word length of words used per valence category.

\begin{tabular}{llllll}
\hline Test phase & Valence & Affect & Familiarity & Affect extremity & Word length (characters) \\
\hline EC & Positive & $6.02(0.32)$ & $5.06(0.59)$ & $2.02(0.32)$ & $5.60(1.26)$ \\
& Negative & $1.95(0.35)$ & $4.86(0.35)$ & $2.06(0.35)$ & $5.30(1.06)$ \\
APT & Neutral & $4.03(0.09)$ & $4.85(0.67)$ & $0.08(0.06)$ & $5.40(1.35)$ \\
& Positive & $5.99(0.24)$ & $6.02(0.29)$ & $1.99(0.24)$ & $7.50(2.35)$ \\
& Negative & $2.02(0.26)$ & $6.01(0.34)$ & $1.98(0.26)$ & $7.75(2.31)$
\end{tabular}

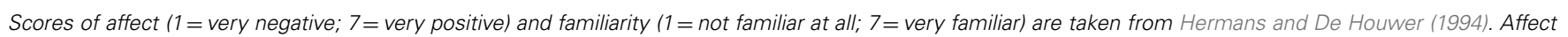
extremity = extent to which the scores deviated from neutral (score 4); EC, evaluative conditioning phase; APT, affective priming task. 
sounds that had been sub-optimally conditioned, one positive and one negative pre-rated sound) were presented with each of the 12 positive targets and with each of the 12 negative targets. The order of the trials was randomized. An obligatory break of at least $3000 \mathrm{~ms}$ was inserted halfway through the task. In order to get used to the task and to the target words the participants carried out 24 practice trials in which all target words were presented once. During practice, feedback was provided on the screen after the trials in which the participants did not respond within $2000 \mathrm{~ms}$ after target onset ("try to respond faster!") or when they incorrectly judged the valence of the target word ("alas, wrong response!"). No feedback was provided during the actual APT.

Post EC subjective valence rating phase. After completing the APT participants again listened to the six CS sounds and rated after each sound how pleasant they experienced it by clicking on nine-point SAMv scale. Beforehand it was stressed that they should rate their current experience and that this might have changed or might have remained the same as compared to their previous rating. Further instructions were similar to those for the ratings in the CS Selection phase.

\section{RESULTS}

Inspection of Boxplots of the RTs per person per condition of the APT revealed that one participant responded consistently slower than the others [above 1.5 inter quartile range criterion in 3 (all consisting of congruent trials) out of 16 conditions]. Therefore the data of this participant were discarded from all analyses.

Unless indicated differently, all performed analyses were repeated measures analyses of variance (ANOVA) or paired $t$ tests. For all analyses a significance level of $\alpha=0.05$ was used. In case of violation of the sphericity assumption, the degrees of freedom were corrected using the Greenhouse-Geisser procedure. With regard to the APT results, trials with $\mathrm{RT}>2000 \mathrm{~ms}(0.6 \%)$ were discarded from analyses. RTs of trials on which the participant incorrectly judged the valence of the target word (2.9\%) were excluded as well.

\section{APT validation with pre-rated primes}

Table 2 shows performance scores for the APT trials with the pre-rated primes. An ANOVA on mean RT with the factors Congruence (congruent, incongruent) and Target Valence (positive,

Table 2 | Mean (SD) of reaction times (ms) and accuracy (percentage correct) for the APT on the congruent, incongruent, and control trials per level of target valence.

\begin{tabular}{|c|c|c|c|c|c|}
\hline \multirow[t]{3}{*}{ Sound } & \multirow[t]{3}{*}{ Condition } & \multicolumn{4}{|c|}{ Targets } \\
\hline & & \multicolumn{2}{|c|}{ Reaction time } & \multicolumn{2}{|c|}{ Accuracy } \\
\hline & & Negative & Positive & Negative & Positive \\
\hline \multirow[t]{2}{*}{ Pre-rated } & Congruent & 645 (139) & $628(116)$ & $98.2(3.5)$ & $98.6(3.2)$ \\
\hline & Incongruent & 664 (132) & 655 (139) & $98.9(2.9)$ & $96.0(7.1)$ \\
\hline \multirow[t]{3}{*}{ CS } & Congruent & $650(122)$ & 635 (115) & $97.5(5.9)$ & $96.7(6.0)$ \\
\hline & Incongruent & $664(137)$ & 666 (130) & $95.3(8.6)$ & 93.5 (7.9) \\
\hline & Control & $643(126)$ & $623(108)$ & $98.9(2.9)$ & $97.8(5.2)$ \\
\hline
\end{tabular}

negative) revealed a main effect of Congruence, $F(1,22)=5.33$, $\mathrm{MSE}=2503.27, p=0.031, \eta_{\mathrm{p}}^{2}=0.20$, no effect of Target Valence, $F(1,22)=1.22, \mathrm{MSE}=3697.07, p>0.05, \eta_{\mathrm{p}}^{2}=0.05$, and no interaction, $F(1,22)<1$.

An ANOVA of accuracy showed no effect of Congruence, $F(1$, $22)<1$, or Target Valence, $F(1,22)=2.48$, MSE $=14.89, p>0.05$, $\eta_{\mathrm{p}}^{2}=0.10$, nor for an interaction, $F(1,22)=3.04, \mathrm{MSE}=20.11$, $p>0.05, \eta_{p}^{2}=0.12$. This indicates that the effect of congruence on RT cannot be attributed to a speed-accuracy trade-off.

\section{APT with CS as primes}

Mean RT and accuracy on the APT trials with CS as primes are shown in Table 2. An ANOVA on RT with the factors Congruence (congruent, incongruent, neutral) and Target Valence (positive, negative) showed an effect of Congruence, $F(2,44)=6.26$, $\mathrm{MSE}=1982.42, p<0.01, \eta_{\mathrm{p}}^{2}=0.22$, but no effect of Target Valence, $F(1,22)=1.85$, MSE $=2237.03, p>0.05, \eta_{\mathrm{p}}^{2}-0.08$, and there was no interaction, $F(2,44)<1$. As expected, responses were slower in the incongruent than in the congruent, $t(22)=2.08$, $p=0.05$, and the neutral condition, $t(22)=3.23, p<0.01$. However, responses were not faster in the congruent than in the neutral condition, $t(22)=1.46, p>0.05$.

An ANOVA of accuracy revealed a main effect of Congruence, $F(2,44)=5.69, \mathrm{MSE}=33.82, . p<0.01, \eta_{\mathrm{p}}^{2}=0.21$, reflecting less correct trials in the incongruent than in the neutral, $t(22)=-3.15$, $p<0.01$ and the congruent condition, $t(22)=-2.00, p=0.06$, but no difference between congruent and neutral trials, $t(22)=-1.32$, $p>0.05$. There was neither a main effect of Target Valence, $F(1$, $22)=1.43, \mathrm{MSE}=35.75, p>0.05, \eta_{\mathrm{p}}^{2}=0.061$, nor an interaction, $F(2,44)<1$. This indicates that the effects of congruence cannot be attributed to a speed-accuracy trade-off.

A separate ANOVA without neutral trials, with Congruence (congruent, incongruent) and Prime Valence (positive, negative) as factors showed an effect of congruence on RT, $F(1$, $22)=4.30, \mathrm{MSE}=2663.35, p<0.05, \eta_{\mathrm{p}}^{2}=0.16$, but no effect of Prime Valence, $F(1,22)<1$, or an interaction, $F(1,22)<1$. That is, conditioning effects occurred for both negative and positive conditioned sounds.

Another separate ANOVA was carried out without neutral trials, but including the trials with pre-rated primes, with Congruence (congruent, incongruent), Target Valence (positive, negative), and Prime Type (CS, pre-rated) as factors. This showed an effect on RT of Congruence $F(1,22)=7.53, \mathrm{MSE}=3286.06, p<0.05$, $\eta_{\mathrm{p}}^{2}=0.21$, but no differences between trials with positive and negative targets $F(1,22)=1.68, \mathrm{MSE}=4724.11, p>0.05, \eta_{\mathrm{p}}^{2}=0.071$, and no difference between trials with pre-rated primes and CS primes $F(1,22)<1$, nor any interaction all $F(1,22)<1$. The absence of an interaction between Congruence and Prime Type shows that effects of Congruence did not differ between trials with pre-rated primes and CS primes.

\section{Subjective valence rating}

In addition to the implicit measure of the conditioning effects, it was investigated whether conditioning was also reflected in subjective ratings. The subjective ratings for conditioned sounds depended on the interaction of the moment of measurement 
(baseline, post EC) and US valence (positive, negative, neutral), $F(2,44)=5.04, \mathrm{MSE}=1.18, p=0.011, \eta_{\mathrm{p}}^{2}=0.19$. At baseline (CS selection phase), ratings did not differ between the sounds that were to be paired with positive $(M=5.00)$, negative $(M=5.09)$, or neutral $(M=5.00)$ words, $F(2,44)<1$. However, after the EC procedure there was a difference of negative $(M=4.09)$ vs. neutral conditions $(M=5.17), t(22)=-3.22, p<0.01$. There was no difference of positive $(M=5.30)$ vs. neutral conditions, $t(22)=0.25$, $p=0.806$.

\section{DISCUSSION EXPERIMENT 1}

In Experiment 1 affectively neutral environmental sounds were paired with positive, negative, or neutral words according to a standard EC procedure. After this procedure the affective value of the conditioned sounds was measured by means of an APT, in which the CS served as prime, and by subjective rating. Because this was the first study to use environmental sounds as primes in an APT, validation trials with pre-rated positive and negative sounds as primes were included to check whether priming effects occurred with the current task parameters. Affective priming effects were found on these trials as expected. This indicates that the APT as used in the current study, is a valid measure of sound evaluation (De Houwer et al., 2009).

Changes in evaluation due to the conditioning procedure were indeed reflected as an interference effect in the APT. Responses to targets preceded by affectively incongruent CS primes were slower than to targets preceded by affectively congruent or neutral CS primes. This demonstrates that the affective value did change due to the EC procedure and that these changes were automatically reflected in behavior. This was the case for both the positive and negative conditioned sounds. Furthermore, there was no difference in the priming effect between trials with CS as primes and pre-rated sounds as primes, which indicates that recently acquired evaluation of environmental sounds is equally strongly reflected in behavior as well-established evaluations. The subjective ratings reflected the $\mathrm{EC}$ effect for sounds paired with negative words only.

Taken together, these results indicate that the affective value of short environmental sounds can be changed in negative direction, and to a lesser extent in positive direction through EC.

\section{EXPERIMENT 2: EXTINCTION}

The first study demonstrated that the affective value of environmental sounds can be changed by means of an EC procedure. A second important issue is whether these changes remain stable over repeated presentations of the conditioned sound alone, that is, whether extinction occurs.

Different theoretical notions of EC generate different predictions about the impact of extinction procedures on the (magnitude of) the acquired evaluation. For example, it has been argued (Baeyens et al., 1992, 2009), that EC is a form of referential learning in which associations are formed automatically as a result of the co-occurrence of the CS and US. After the association has been established, activation of the CS will activate a representation of the US which, unlike in other forms of conditioning, is not accompanied by the expectancy that the US will occur. Since it is not the expectancy of the US occurrence that generates the evaluative response to the CS, but the mere association with the US, EC should be resistant to extinction procedures. Repeated presentation of the CS without a subsequent US should thus leave the acquired evaluation unaffected (Díaz et al., 2005).

Opposite predictions are made by accounts that propose that the process underlying EC is the same as the process underlying (other forms of) Pavlovian Conditioning (Lipp et al., 2003; Lipp and Purkis, 2005, 2006). Pavlovian Conditioning is generally regarded as a form of signal learning in which propositions are formed about CS-US contingencies. After conditioning, the CS signals the occurrence of the US and the CS is evaluated according to the valence of the US. During extinction the propositions about CS-US contingency are updated and CS evaluation changes accordingly (De Houwer et al., 2005; De Houwer, 2007). These accounts do not predict that EC is resistant to extinction (Lipp and Purkis, 2005).

So far studies examining the role of extinction in EC have yielded mixed results, several studies report that the evaluation of the CS remains stable after an extinction procedure (Baeyens et al., 1988; Díaz et al., 2005), while others found that the evaluations returned to neutral during extinction (Lipp et al., 2003; Lipp and Purkis, 2006). A recent meta-analysis suggests that the EC effect does not disappear after repeated presentation, but does decrease compared to the effect measured directly after conditioning (Hofmann et al., 2010).

Proponents of the single process account for EC and PC have suggested that findings of resistance to extinction can be attributed to the way in which ratings of the evaluations of the CS were obtained (Lipp and Purkis, 2006). In most studies that found resistance to extinction, CS ratings were collected only at the end of the acquisition and extinction phase of the experiment. According to Lipp and Purkis (2006), when judgments are obtained at the end of the extinction phase only, instead of in the course of the whole experiment, people tend to integrate information about the CS across the acquisition and extinction phases. Therefore these judgments may not reflect actual current stimulus evaluation.

Given the unresolved issues concerning the process of EC and its resistance to extinction it is important to thoroughly investigate whether the evaluation acquired during conditioning remains stable over repeated presentations of the conditioned sound alone. Therefore, in the second study we used a conditioning procedure followed by a substantial number of extinction trials while repeatedly asking people for their judgments.

As in Experiment 1, the experimental procedure of Experiment 2 consisted of several phases. The CS selection phase and conditioning phase were identical to those in the first study, except for an additional CS rating after 10 CS-US parings. The conditioning phase was followed by an extinction phase in which the CS were presented without the US. Ratings of the CS during the extinction phase were acquired after every 10 presentations of all conditioned sounds. Given the conflicting findings from previous studies with respect to the effect of extinction procedures, we had no clear expectations about the stability of the acquired evaluations of the CS during and after extinction. 


\section{MATERIALS AND METHODS \\ Participants}

Twenty-four Dutch speaking participants (four male, two left handed) with self-reported normal hearing and ranging in age from 18 to 30 years ( $M=20.9$ years, $\mathrm{SD}=3.3$ years) took part in the experiment for which they received course credits or $€ 6.50$. All participants signed an informed consent before the start of the study. The study was approved by the ethical committee of the Institute of Psychology of Leiden University.

\section{Materials}

Apparatus, sounds, and words for the conditioning phase were identical to those of Experiment 1.

\section{Procedure}

Up to the EC phase the procedure was identical to the first experiment, with the exception that participants were asked to rate the pleasantness of each CS on the nine-point SAMv scale after each CS had been paired 10 times with an US. Instructions for CS rating were the same as in study one. The CS rating was repeated after another 10 CS-US pairings for each CS. This was followed by an assessment of contingency awareness to assess the differences in awareness of CS-US contingencies between EC with sub-optimally and supra-optimally presented US words, which will not be discussed in the current paper.

\section{Extinction phase}

After the contingency awareness assessment participants were told that they would again hear the sounds repeatedly. They were instructed to concentrate on the sounds and they were informed that they would be asked to rate the sounds four times during this part of the experiment. Sound ratings were collected in the same manner as during the Conditioning phase. In total there were six moments of measurement (MM) during the conditioning and acquisition phases (see Figure 1). The last rating was again followed by a contingency awareness assessment.

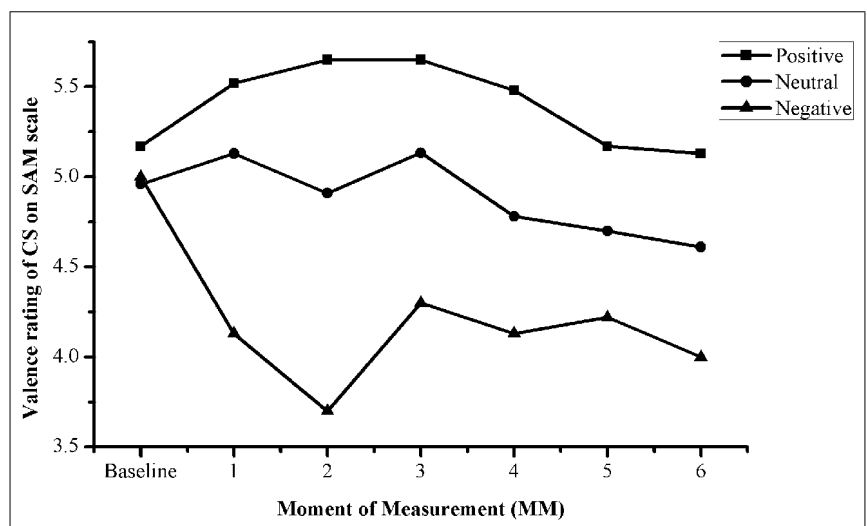

FIGURE 1 | Mean of ratings on the nine-point SAMv scale per moment of measurement for CS sounds that were paired with positive, negative, or neutral US words at different moments of measurement (MM). MM 1 and 2 are after 10 and 20 CS-US parings, respectively. MM 3, 4,5 , and 6 are after 10, 20, 30, and 40 extinction trials, respectively.

\section{RESULTS}

Inspection of Boxplots of the SAMv ratings per person per CS per MM revealed that one participant rated all sounds as very unpleasant at baseline and these ratings exceeded the three inter quartile range criterion. Therefore the data of this participant were excluded from analysis.

Unless indicated differently, all performed analyses were repeated measures ANOVAs or paired $t$-tests. For all analyses a significance level of $\alpha=0.05$ was used. In case of violation of the sphericity assumption, the degrees of freedom were corrected using the Greenhouse-Geisser procedure.

\section{Evaluative conditioning effect}

Firstly it was tested whether the EC effect occurred looking at the baseline CS rating and the CS rating at the end of the conditioning phase. The ratings depended on the interaction of MM (baseline vs. MM2) and Valence (positive, negative, neutral), $F(2,44)=6.89, \mathrm{MSE}=1.40, p<0.01, \eta_{\mathrm{p}}^{2}=0.24$. At baseline (CS selection phase) ratings did not differ between the sounds that were to be paired with positive $(M=5.17)$, negative $(M=5.00)$, or neutral $(M=4.96)$ words, $F(2,44)<1$. However the ratings of these sounds did differ on the measurements after the EC procedure, $F(2,44)=8.63, \mathrm{MSE}=2.60$, $p<0.01, \eta_{\mathrm{p}}^{2}=0.28$. Just as in Experiment 1 , the sounds paired with the negative US words were rated more negatively after conditioning $(M=3.70)$ than the sounds paired with the neutral US words $(M=4.91), t(22)=-2.421, p=0.02$. Sounds paired with positive US words were rated marginally more positively than sounds paired with neutral words, $t(22)=-1.95$, $p=0.06$.

\section{Extinction}

Figure 1 shows the subjective ratings of CS as a function of MM. An ANOVA of the ratings was performed with the factors Valence (positive, negative, neutral) and MM (MM2, MM3, MM4, MM5, and MM6). While there was an effect of valence, $F(2,44)=10.58, p<0.01, \eta_{\mathrm{p}}^{2}=0.33$, there was no main effect of $\mathrm{MM}, F(4,88)<1$, and no interaction of $\mathrm{MM}$ and valence, $F_{\mathrm{GG}}(8$, $176)=1.034, \mathrm{MSE}=2.03, p=0.39, \eta_{\mathrm{p}}^{2}=0.05$. This indicates that the EC effect did not change over time, which implies that no extinction occurred.

However, given the fact that null results do not allow strong conclusions, the rating pattern at the end of the extinction phase (MM6) was examined separately as a stricter test for resistance to extinction. This concerned the same contrast as used to test for the conditioning effect at MM2. At MM6 the sounds paired with the negative US words were not rated significantly more negative after conditioning $(M=4.00)$ than the sounds paired with the neutral US words $(M=4.61), t(22)=-1.39, p=0.18$, likewise sounds paired with positive words $(M=5.13), t(22)=1.78$, $p=0.09$, were not rated significantly more positive than sounds paired with neutral words. Furthermore, the effect sizes (Cohen's d) for sounds paired with negative words (vs. neutral) decrease from $d=0.82$ at MM2 to $d=0.39$ at MM6, and for the positive (vs. neutral) from $d=0.50$ at MM2 to $d=0.39$ at MM6. However, there was still a difference between the sounds with negative words compared to sounds paired with positive words as indicated by the 
effect of US valence in the overall analysis at MM6, $F(2,44)=4.19$, $\mathrm{MSE}=1.76, p=0.02, \eta_{\mathrm{p}}^{2}=0.16$.

\section{DISCUSSION EXPERIMENT 2}

In Experiment 2 it was investigated to what extent the EC effect for environmental sounds was affected by an extinction procedure. To this end a conditioning procedure was followed by a substantial number of extinction trials, while repeatedly asking people to rate the affective valence of the sounds. Effects of conditioning were indeed found in the subjective ratings of the sounds paired with negative words and to a lesser extent in the subjective ratings of the sounds paired with positive word. At first sight these effects did not change with repeated presentation of the sounds in the absence of the US, suggesting resistance to extinction.

However, further analysis suggested that the EC effect as reflected in the subjective ratings were more apparent at the end of the conditioning period than at the end of the extinction period. This extinction effect is in line with the general pattern in previous EC studies. Hofmann et al. (2010) concluded that while the effects of EC are still present after extinction, the magnitude of the effect decreased from post acquisition to post extinction.

\section{GENERAL DISCUSSION}

To our knowledge the present study is the first to demonstrate changes in affective value of short environmental sounds through an EC procedure. The first experiment showed that EC effects were reflected in an explicit (subjective) as well as an implicit (RT and error rate) measure. The second experiment indicated that the acquired affective value, albeit weaker, remains present over a substantial number of repeated presentations of the CS alone.

The first experiment showed that the effects of the EC procedure evoked genuine changes in affective evaluation of environmental sounds. The changes in evaluation due to the conditioning procedure were reflected as an interference effect in the APT. Given that responses on the APT are low in controllability (De Houwer, 2003; Gawronski, 2009), it is unlikely that the effects of the EC procedure are attributable to demand effects (Hermans et al., 2003b). Furthermore, the effect in the APT indicates that the acquired evaluation can behaviorally manifest itself in an automatic way (De Houwer et al., 2009). It has been argued that effects on implicit measures such as the APT mimic spontaneous behavior in real life (Fazio and Olson, 2003; De Houwer, 2006). Therefore these findings suggest that learned evaluation of environmental sounds could influence spontaneous behavior in our daily lives.

The effects on the APT also demonstrate that the affective value can be changed through EC in negative as well as positive direction. That is, sounds paired with positive US words during the conditioning phase that were used as prime in the APT decreased response times to negatively valenced target words and vice versa. However, the subjective ratings in the first study only reflected effects of conditioning for the sounds paired with negative words compared to sounds paired with neutral words, while ratings of sounds paired with positive words did not differ from the latter. This might suggest the presence of a negativity bias, the idea that negative events are more salient, powerful, and have stronger effects compared to positive events (Kanouse and Hanson, 1972; Rozin and Royzman, 2001). Indeed several previous studies did find stronger EC effects on explicit ratings for stimuli paired with negative than for positive effects (e.g., in the gustatory domain; Baeyens et al., 1990).

Differences between positive and negative conditioned stimuli might also arise when the degree to which the negative US are evaluated as negative is larger than the degree to which the positive US are evaluated as positive. In the present study positive and negative US were matched on affective extremity based on norms of a Flemish study (Hermans and De Houwer, 1994) which are likely to be applicable for Dutch participants. Future studies, however could include individual ratings of the US to control even more for affective extremity differences. Differences in individual US ratings may explain that, while the subjective conditioning effect for sounds paired with positive words was absent in the first experiment, there was a small effect in the second experiment.

The second experiment showed that effects of EC are still present after at least $40 \mathrm{CS}$ presentations in absence of the US. However, the effects were less pronounced after the extinction phase compared to directly after conditioning. Given the prediction of resistance to extinction (Baeyens et al., 1992), the findings cannot be fully explained by the referential account of EC which assumes that the EC effect occurs due to automatically formed CS-US associations. However, the data do also not fully reflect expectancy learning as in Pavlovian Conditioning in which propositions are formed about CS-US contingencies (Lipp and Purkis, 2006). Lipp and Purkis (2006) argued that CS evaluations show extinction, but at a slower rate than measures of US expectancy ratings. Previous studies claiming resistance to extinction have been criticized because too few extinction trials were used to demonstrate extinction as reflected in subjective evaluation of the CS (Lipp and Purkis, 2006). However, in the present study, even after a substantial number (40) of extinction trials per CS, the effect still remained. Furthermore, it is unlikely that the EC effect in the final CS ratings reflect an integration of information about both the acquisition and extinction phase as is the case when only post-experimental ratings are taken. In the current study, repeated measurements of CS evaluation were taken throughout acquisition and extinction phases. Repeated measurements throughout the experiment have been shown to reflect current valence ratings rather than ratings based on integration of information over the experiment (Lipp and Purkis, 2006).

The present pattern of results best fits a dual process account of EC, which suggests that EC effects can occur through both referential and propositional processes (De Houwer et al., 2005; De Houwer, 2007). If these processes do occur simultaneously then part of the EC effect will disappear during extinction due to changes in US expectancy, while the EC effect formed through associations by mere co-occurrence of CS and US during the acquisition phase will remain after extinction. The current paradigm using explicit valence assessment and including a measure of contingency awareness prior to the extinction procedure may have emphasized propositional information about the CS and US contingencies. To avoid these effects a follow-up study may intermix an EC and extinction procedure with APT trials, through which a more unobtrusive measurement of valence can be obtained.

Taken together, the results of our study demonstrate that through EC short environmental sounds can attain a negative or, 
to a lesser extent, a positive value. This effect is reflected in both implicit and explicit measures, and persists, although in decreased magnitude, after repeated presentation of the sound. These findings are best explained by a dual-processing account of EC in which associative as well as propositional processes underlie the EC effect.

Our demonstration of EC in the auditory domain may further advance research into affective sound processing. Studying the influence of affective valence on auditory processing is hampered by confounds due to differences in acoustical features inherent to positive negative and neutral sounds (Aeschlimann et al., 2008). In addition, the study of affective sound processing using event-related brain potentials would only be possible with short sounds, so that the relevant information becomes available with a more or less constant timing. This requirement is hard to meet with naturalistic affective sounds. As we have shown, EC makes it possible to change the valence of sounds.

\section{REFERENCES}

Aeschlimann, M., Knebel, J. F., Murray, M., and Clarke, S. (2008). Emotional pre-eminence of human vocalizations. Brain Topogr. 20, 239-248.

Aguado, L., Manuel, P., and Cristina, S. (2005). Affective priming with associatively acquired valence. Psicológica 26, 261-279.

Baeyens, F., Crombez, G., Van Den Bergh, O., and Eelen, P. (1988). Once in contact always in contact: evaluative conditioning is resistant to extinction. Adv. Behav. Res. Ther. 10, 179-199.

Baeyens, F., Eelen, P., Crombez, G., and Van Den Bergh, O. (1992). Human evaluative conditioning: acquisition trials, presentation schedule, evaluative style and contingency awareness. Behav. Res. Ther. 30, 133-142.

Baeyens, F., Eelen, P., Van Den Bergh, O., and Crombez, G. (1990). Flavorflavor and color-flavor conditioning in humans. Learn. Motiv. 21, 434-455.

Baeyens, F., Vansteenwegen, D., and Hermans, D. (2009). Associative learning requires associations, not propositions. Behav. Brain Sci. 32, 198-199.

Bliss-Moreau, E., Owren, M. J., and Barrett, L. F. (2010). I like the sound of your voice: affective learning about vocal signals. J. Exp. Soc. Psychol. 46, 557-563.

Bradley, M. M., and Lang, P. J. (1994). Measuring emotion: the selfassessment manikin and the semantic differential. J. Behav. Ther. Exp. Psychiatry 25, 49-59.

Bradley, M. M., and Lang, P. J. (2007). The International Affective Digitized Sounds (IADS-2): Affective Ratings of Sounds and Instruction Manual, 2nd Edn, Technical Report
B-3. Gainesville, FL: University of Florida.

De Houwer, J. (2003). "A structural analysis of indirect measures of attitudes," in The Psychology of Evaluation. Affective Processes in Cognition and Emotion, eds J. Musch and K. C. Klauer (Mahwah, NJ: Lawrence Erlbaum Associates), 219-244.

De Houwer, J. (2006). "What are implicit measures and why are we using them," in The Handbook of Implicit Cognition and Addiction, eds R. W. Wiers and A. W. Stacy (Thousand Oaks, CA: Sage Publishers), 11-28.

De Houwer, J. (2007). A conceptual tive conditioning. Span. J. Psychol. 10, 230-241.

De Houwer, J. (2008). "Conditioning as a source of liking: there is nothing simple about it," in Frontiers of Social Psychology: The Social Psychology of Consumer Behavior, ed. M. Wänke (New York: Psychology Press), 151-166.

De Houwer, J., Baeyens, F., and Field, A. (2005). Associative learning of likes and dislikes: some current controversies and possible ways forward. Cogn. Emot. 19, 161-174.

De Houwer, J., Teige-Mocigemba, S., Spruyt, A., and Moors, A. (2009). Implicit measures: a normative analysis and review. Psychol. Bull. 135, 347-368.

De Houwer, J., Thomas, S., and Baeyens, F. (2001). Associative learning of likes and dislikes: a review of 25 years of research on human evaluative conditioning. Psychol. Bull. 127, 853-869.

Degner, J. (2008). On the (un)controllability of affective priming: strategic manipulation is feasible but can possibly be prevented. Cogn. Emot. 23, 327-354. and theoretical analysis of evalua-

EC therefore enables studying short acoustically identical stimuli that have acquired different affective valence for different people, and facilitates an event-related potentials approach. From our finding of partial resistance to extinction it follows that this method can also be used if repeated presentation of CS sounds in absence of the US is required. Thus, the current study not only demonstrates that the EC effect generalizes to the auditory domain, it also paves the way to study affective environmental sound processing while effectively controlling acoustic properties.

\section{ACKNOWLEDGMENTS}

This research was financed in part by Platform Duurzame Luchtvaart (PDL, Platform Sustainable Aviation), The Netherlands. The authors would like to thank Floor Baten, Nadia den Braber, Linda Couwenberg, Britt Dijkstra, and Germaine Posman for their assistance in data collection.

Díaz, E., Ruiz, G., and Baeyens, F. (2005). Resistance to extinction of human evaluative conditioning using a between-subjects design. Cogn. Emot. 19, 245-268.

Fabiani, M., Kazmerski, V. A., Cycowicz, Y., and Friedman, D. D. (1996). Naming norms for brief environmental sounds: effects of age and dementia. Psychophysiology 33, 462-475.

Fazio, R. H., and Olson, M. A. (2003). Implicit measures in social cognition research: their meaning and use. Annu. Rev. Psychol. 54, 297-327.

Field, A. P. (2005). "Learning to like (or dislike): associative learning of preferences," in New Directions in Human Associative Learning, ed. A. J. Wills (Mahwah, NJ: Lawrence Erlbaum Associates), 221-252.

Gawronski, B. (2009). Ten frequently asked questions about implicit measures and their frequently supposed, but not entirely correct answers. Can. Psychol. 50, 141-150.

Gygi, B., Kidd, G. R., and Watson, C. S. (2007). Similarity and categorization of environmental sounds. Percept. Psychophys. 69, 839-855.

Hermans, D., Baeyens, F., and Eelen, P. (2003a). "On the acquisition and activation of evaluative information in memory: the study of evaluative learning and affective priming combined," in The Psychology of Evaluation. Affective Processes in Cognition and Emotion, eds J. Musch and K. C. Klauer (Mahwah, NJ: Lawrence Erlbaum Associates), 139-168.

Hermans, D., Spruyt, A., and Eelen, P. (2003b). Automatic affective priming of recently acquired stimulus valence: priming at SOA 300 but not at SOA 1000. Cogn. Emot. 17, 83-99.

Hermans, D., De Houwer, J., and Eelen, P. (2001). A time course analysis of the affective priming effect. Cogn. Emot. 15, 143-165.

Hermans, D. D., and De Houwer, J. (1994). Affective and subjective familiarity ratings of 740 Dutch words. Psychol. Belg. 34, 115-139.

Hofmann, W., De Houwer, J., Perugini, M., Baeyens, F., and Crombez, G. (2010). Evaluative conditioning in humans: a meta-analysis. Psychol. Bull. 136, 390-421.

Kanouse, D. E., and Hanson, L. (1972). "Negativity in evaluations," in Attribution: Perceiving the Causes of Behavior, eds E. E. Jones, D. E. Kanouse, S. Valins, H. H. Kelley, R. E. Nisbett, and B. Weiner (Morristown, NJ: General Learning Press), 47-62.

Levey, A. B., and Martin, I. (1975). Classical conditioning of human "evaluative" responses. Behav. Res. Ther. 13, 221-226.

Lipp, O. V., Oughton, N., and Lelievre, J. (2003). Evaluative learning in human Pavlovian conditioning: extinct, but still there? Learn. Motiv. 34, 219-239.

Lipp, O. V., and Purkis, H. M. (2005). No support for dual process accounts of human affective learning in simple Pavlovian conditioning. Cogn. Emot. 19, 269-282.

Lipp, O. V., and Purkis, H. M. (2006). The effects of assessment type on verbal ratings of conditional stimulus valence and contingency judgments: implications for the extinction of evaluative learning. J. Exp. Psychol. Anim. Behav. Process 32, 431-440.

Mitchell, C. J., Anderson, N. E., and Lovibond, P. F. (2003). Measuring evaluative conditioning using the implicit association test. Learn. Motiv. 34, 203-217. 
Orne, M. T. (1962). On the social psychology of the psychological experiment: with particular reference to demand characteristics and their implications. Am. Psychol. 17, 776-783.

Rozin, P., and Royzman, E. B. (2001). Negativity bias, negativity dominance, and contagion. Pers. Soc. Psychol. Rev. 5, 296-320.

Schneider, W., Eschman, A., and Zuccolotto, A. (2002). E-Prime User's
Guide. Pittsburgh: Psychology Software Tools Inc.

Teige-Mocigemba, S., and Klauer, K. C. (2008). "Automatic" evaluation? Strategic effects on affective priming. J. Exp. Soc. Psychol. 44, 1414-1417.

Zajonc, R. B. (1968). Attitudinal effects of mere exposure. J. Pers. Soc. Psychol. 9(Pt 2), 1-27.

Zajonc, R. B. (1980). Feeling and thinking: preferences need no inferences. Am. Psychol. 35, 151-175.
Conflict of Interest Statement: The authors declare that the research was conducted in the absence of any commercial or financial relationships that could be construed as a potential conflict of interest.

Received: 13 January 2012; accepted: 23 March 2012; published online: 10 April 2012.

Citation: Bolders AC, Band GPH and Stallen PJ (2012) Evaluative conditioning induces changes in sound valence. Front. Psychology 3:106. doi: 10.3389/fpsyg.2012.00106

This article was submitted to Frontiers in Emotion Science, a specialty of Frontiers in Psychology.

Copyright (C) 2012 Bolders, Band and Stallen. This is an open-access article distributed under the terms of the Creative Commons Attribution Non Commercial License, which permits noncommercial use, distribution, and reproduction in other forums, provided the original authors and source are credited. 


\section{APPENDIX}

Table A1 | Description, length, mean and SD of SAMv rating and source of the sounds used as CS in the evaluative conditioning phase and as primes in the affective priming phase.

\begin{tabular}{|c|c|c|c|c|}
\hline Application & Description & Length (ms) & SAMv $M$ (SD) & Source \\
\hline \multirow[t]{11}{*}{ CS } & Basket ball bouncing & 333 & $5.00(0.93)$ & Gygi et al. (2007) \\
\hline & Zipper & 383 & $5.00(0.85)$ & \\
\hline & Chicken cackling & 375 & $4.93(1.44)$ & \\
\hline & Mallard quacking & 204 & $5.07(2.05)$ & \\
\hline & Seal barking & 400 & $5.27(1.53)$ & \\
\hline & Wolf growling & 391 & $4.73(1.44)$ & \\
\hline & Raven cawing & 215 & $4.73(1.44)$ & \\
\hline & Car starting & 216 & $4.67(1.29)$ & Gygi et al. (2007) \\
\hline & Pig grunting & 215 & $5.40(1.68)$ & Fabiani et al. (1996) \\
\hline & Sneeze & 333 & $4.20(1.37)$ & Gygi et al. (2007) \\
\hline & Water pouring & 392 & $5.93(1.16)$ & \\
\hline \multirow[t]{2}{*}{ Pre-rated } & Bird chirping & 346 & $6.87(1.85)$ & Bradley and Lang (2007) \\
\hline & Gun shot & 343 & $2.87(1.64)$ & Gygi et al. (2007) \\
\hline \multirow[t]{4}{*}{ Practice } & Clock ticking & 142 & $5.13(1.25)$ & \\
\hline & Drum roll & 361 & $5.20(1.61)$ & Fabiani et al. (1996) \\
\hline & Phone ringing (old) & 415 & $5.33(1.61)$ & Gygi et al. (2007) \\
\hline & Helicopter & 297 & $5.53(1.30)$ & \\
\hline
\end{tabular}

All sounds were rated previously in a separate sound rating study on a computer administered pictorial nine-point self assessment-manikin valence scale (SAMv; Bradley and Lang, 1994) ranging from very upleasant (1) to very pleasant (9) (N=15, age: 18-30, normal hearing, Dutch speaking). 
Table A2 | Valence category affect, affect extremity, familiarity, and word length of words used in the evaluative conditioning phase.

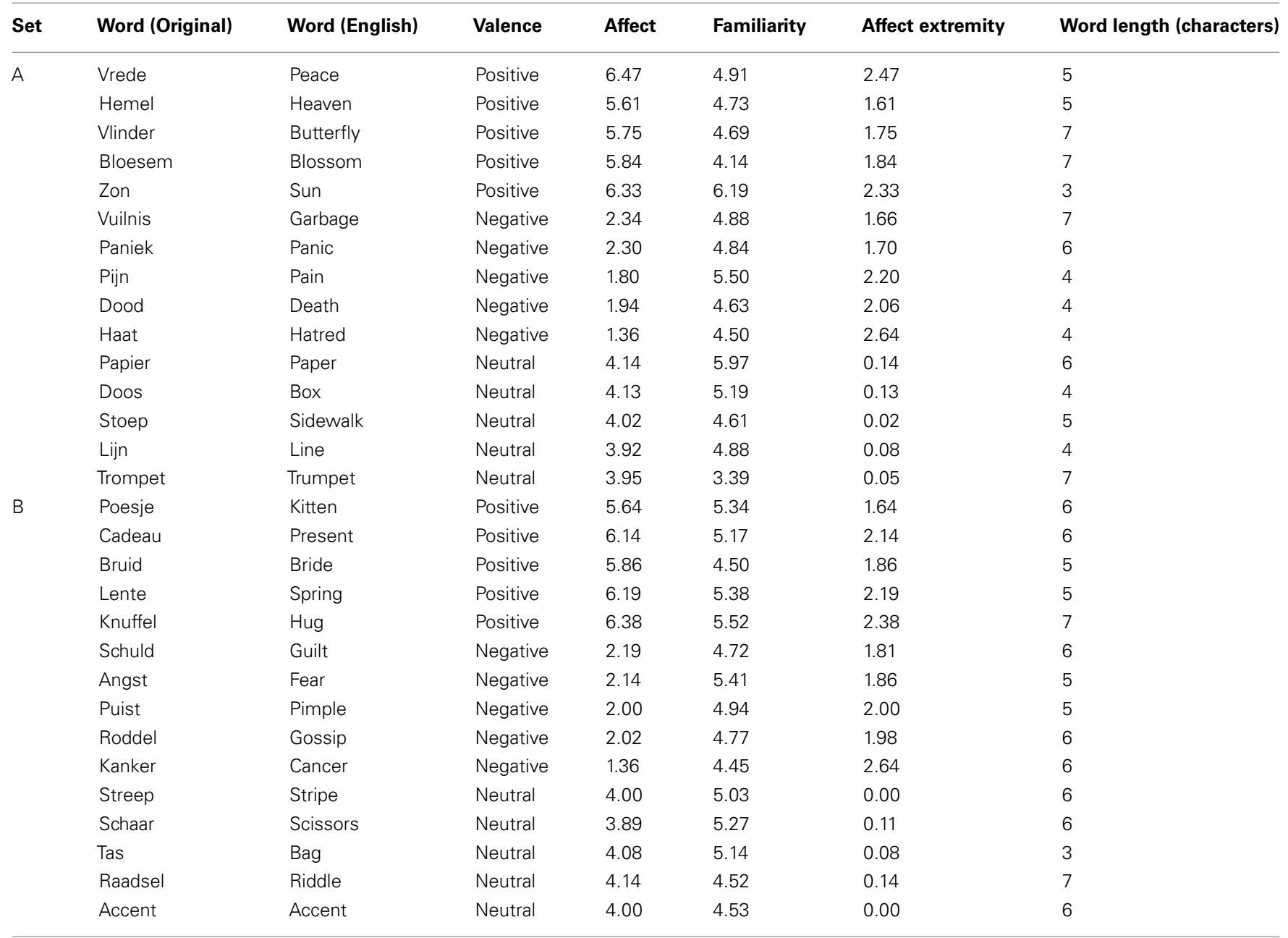

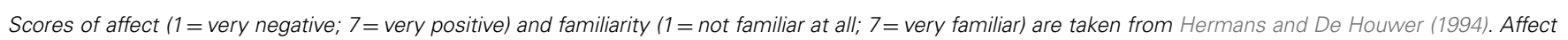
extremity = extent to which the scores deviated from neutral (score 4$)$. 
Table A3 | Valence category affect, affect extremity, familiarity, and word length of words used as targets in the affective priming task.

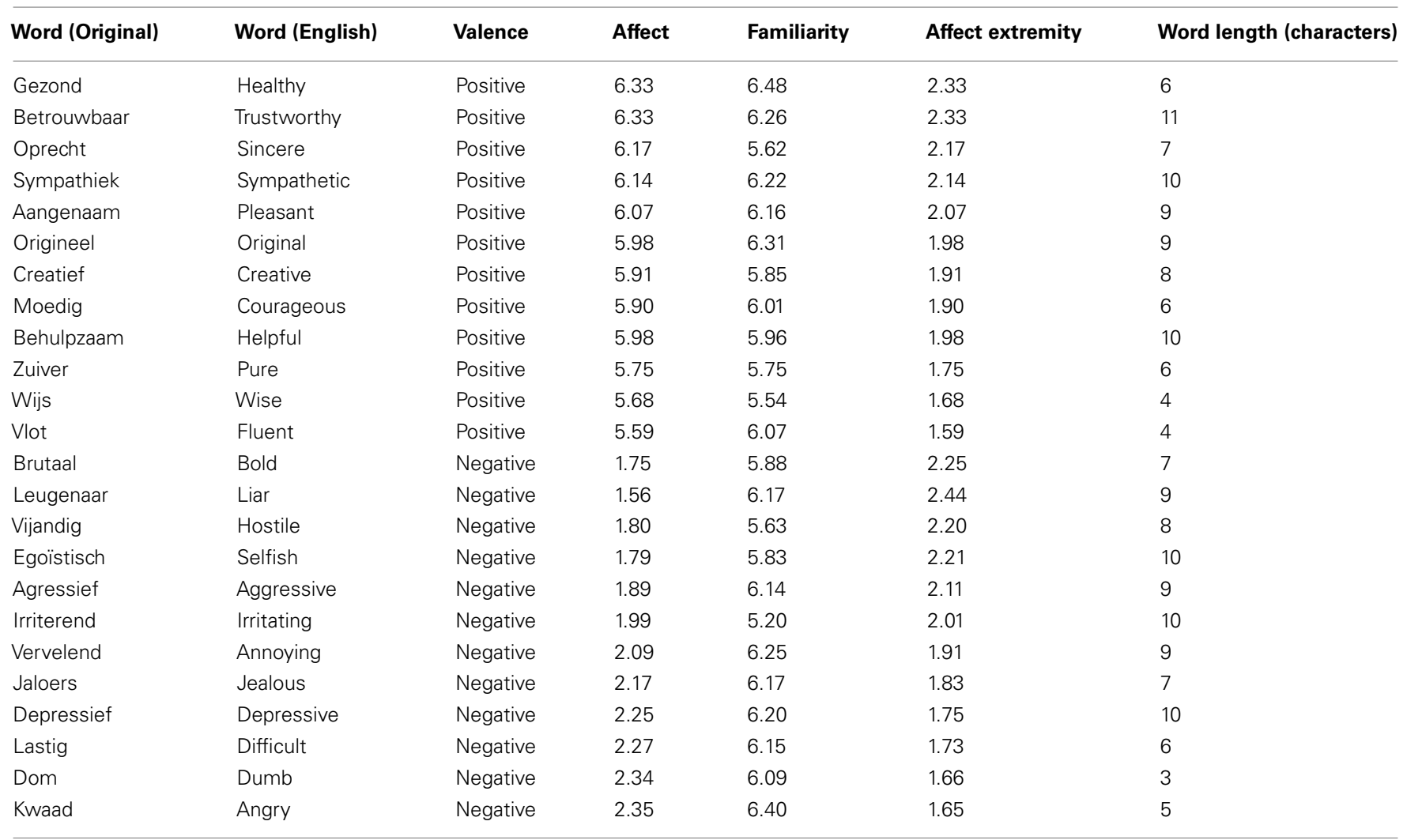

Scores of affect ( $1=$ very negative; $7=$ very positive) and familiarity $(1=$ not familiar at all; $7=$ very familiar) are taken from Hermans and De Houwer (1994). Affect extremity= extent to which the scores deviated from neutral (score 4). 